\title{
Patient-Specific Model of Left Heart Anatomy, Dynamics and Hemodynamics from 4D TEE: A First Validation Study
}

Ingmar Voigt ${ }^{1,2, \star}$, Tommaso Mansi ${ }^{1}$, Viorel Mihalef ${ }^{1}$, Razvan Ioan Ionasec ${ }^{1}$, Anna Calleja ${ }^{3}$, Etienne Assoumou Mengue ${ }^{1}$, Puneet Sharma ${ }^{1}$, Helene Houle ${ }^{4}$, Bogdan Georgescu ${ }^{1}$, Joachim Hornegger ${ }^{2}$, and Dorin Comaniciu ${ }^{1}$

1 Image Analytics and Informatics, Siemens Corporate Research, Princeton, NJ, USA ingmar.voigt. ext@siemens.com

2 Pattern Recognition Lab, Friedrich-Alexander-University, Erlangen, Germany

3 Davis Heart and Lung Research Institute, Ohio State University, Columbus, OH, USA

4 Ultrasound, Siemens Healthcare, Mountain View, CA, USA

\begin{abstract}
Patient-specific models of the heart physiology have become powerful instruments able to improve the diagnosis and treatment of cardiac disease. A systemic representation of the whole organ is required to capture the complex functional and hemodynamical interdependencies among the anatomical structures. We propose a novel framework for personalized modeling of the left-side heart that integrates comprehensive data of the morphology, function and hemodynamics. Patient-specific fluid dynamics are computed over the entire cardiac cycle using embedded boundary and ghost fluid methods, constrained by the dynamics of highly detailed anatomical models. Personalized boundary conditions are determined by estimating cardiac shape and motion from 4D TEE images through robust discriminative learning methods. Qualitative and quantitative validation of the computed blood dynamics is performed against Doppler echocardiography measurements, following an original methodology. Results showed a high agreement between simulation and ground truth and a correlation of $r=0.85(p<0.0002675)$. To the best of our knowledge, this is the first time that computational fluid dynamics are simulated on a systemic and comprehensive patient-specific model of the heart and validated against routinely acquired clinical ground truth.
\end{abstract}

\section{Introduction}

Cardiovascular disease management is nowadays largely supported by increasingly more accurate, fast and ubiquitous imaging technologies. However, this rich information is barely exploited in the clinical decision making process. Nonrespondent patients are common in cardiac disease [1, presenting in numerous cases unexpected adverse events because the therapy was not adapted to that

\footnotetext{
^ Corresponding author.
} 
specific patient. In fact therapeutical decisions are largely based on results obtained in population-wise studies and are therefore not personalized. Furthermore, the complex interdependency of anatomy, function and hemodynamics imposes the need of a systematic analysis of the whole organ to accurately assess dysfunction and associated morbidities. Therefore, there is a growing need for patient-specific models that 1) provide the cardiologist with accurate, quantitative, and reproducible biomarkers of the cardiac function 2) give insights and predict comorbidites within the complex interconnected cardiovascular system and 3) can predict, beforehand, the outcome of a therapy [2]. These models would enable and support personalized, preventive and predictive healthcare by predicting disease progress and therapeutical outcomes.

In the last decades, tremendous efforts have been made to enable computational fluid dynamics (CFD) in cardiac models in order to integrate into a holistic view the organ anatomy, dynamics an hemodynamics. Due to the lack of personalized representations, fluid dynamics equations are often solved on generic models built from at most one cardiac phase with simplified motion [3. Although patient-specific measurements have been increasingly used to enhanced computational models 45], current simulations are still lacking the accuracy required in the clinical practice. Recently, detailed anatomical models of the heart dynamics have been proposed [6]. Such models, coupled with CFD frameworks [4] open the way to data-driven patient-specific models of anatomy, dynamics and hemodynamics.

In this paper we propose a highly detailed patient-specific model of left-heart anatomy, dynamics and hemodynamics and its validation against clinically acquired Doppler measurements. The contributions are three-fold: i) The first model of left heart with fast and robust patient-specific parameter estimation from four-dimensional transesophageal echocardiography (TEE) (Sec. 2), ii) an improved patient-specific hemodynamics model, computed by solving a level set formulation of the Navier-Stokes equations (Sec. 3) and iii) a qualitative and quantitative validation against clinical Doppler echocardiography (Sec. 4). The results, reported in Sec. 5, demonstrate the validity of our approach.

\section{Patient-Specific Anatomy and Dynamics Computation}

We propose a holistic four-dimensional model of the left heart that comprises its core anatomical structures (Fig. 1): left ventricle (LV), left atrium (LA), aortic valve $(\mathrm{AV})$, mitral valve (MV) and its papillary muscles (APM and PPM). Given the physiological complexity of the left heart, we selected a modular and hierarchical approach, which facilitates capturing a broad spectrum of morphological and pathological variations. The model is parameterized as follows:

1. Four time dependent similarity transforms for each anatomical structure (LV, LA, AV and MV) comprising their global location, orientation and scale over the cardiac cycle, denoted by $B(t)_{m}, m \in\{\mathrm{LV}, \mathrm{LA}, \mathrm{AV}, \mathrm{MV}\}$.

2. 20 trajectories of anatomically defined landmarks $l_{n} \in \mathbb{R}^{3}$ (3 commissures, 3 hinges, 3 tips and 2 ostia for the aortic valve, and 3 trigones, 2 commissures, 2 tips and 2 papillary heads for the mitral valve), $L(B, t)=\left\{l_{1}, l_{2}, \ldots, l_{20}\right\}$. 
3. Nine dense meshes $M_{q}$ with $K_{q}$ vertices to represent the LA, LV, aortic root, three aortic leaflets, two mitral leaflets and the aortic-mitral continuity $M_{q}(B, L, t)=\left\{\mathbf{v}_{1}, \mathbf{v}_{2}, \ldots, \mathbf{v}_{K_{q}}\right\}, v_{i} \in \mathbb{R}^{3}$ being the position of the $i^{t h}$ vertex, which are constrained by the previously defined landmarks.

The patient-specific parameters of the valvular apparatus and left ventricle are estimated from 4D TEE images using a hierarchical discriminative learning algorithm as proposed in [67]. The a posteriori probability $p(B, L, M \mid I)$ of the model given the image data $I$ is incrementally modeled within the Marginal Space Learning (MSL) framework. The similarity transforms $B$ and the anatomical landmarks $L$ are estimated automatically by detectors successively trained on the marginal spaces using the Probabilistic Boosting Tree (PBT) 8 with Haar and steerable features. The complex local motion of the surface structures is estimated with a combination of the aforementioned techniques and speckle tracking methods [7. For further details on model estimation the reader is referred to 677. The left atrium (LA) and pulmonary veins are commonly only partially visible in TEE acquisitions, which hampered an accurate automatic segmentation. Therefore, the LA fitting is performed semi-automatically along the cardiac cycle, using constraints provided by the mitral annulus and statistical models of shape and motion obtained from a large CT database [8].

It is important to note that our model is anatomically highly detailed and considers a number of important aspects for the computation of patient-specific blood flow. Firstly, valve leaflets are volumetrically modeled by representing them with both ventricular and arterial / atrial surface (Fig. 11 Top right). Secondly, the papillary muscles are represented as part of the left ventricular endocardium, constrained by the papillary heads (Fig. 1 Bottom right).

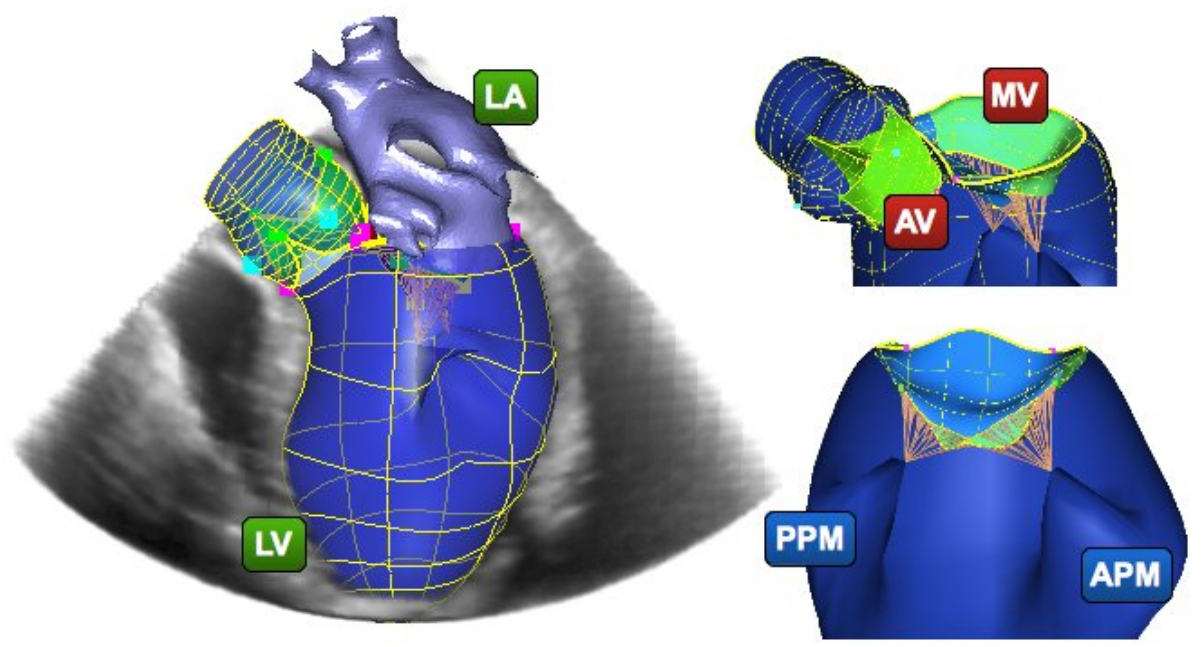

Fig. 1. Proposed model of anatomy and dynamics. Left: full model of the left heart estimated from TEE data. Top right: view of the aortic and mitral valve with volumetric leaflets. Bottom right: septal view of the left ventricular papillary muscles. 
These first two aspects consider their spatial presence and displacement of blood. Thirdly, the model includes the longitudinal, radial and circumferential left ventricular motion to capture the full momentum exercised by the endocardial wall onto the blood [7. Fourthly, the meshes are uniquely parameterized through the anatomical landmarks $l_{n}$. Thereby temporal point correspondence is implicitly guaranteed, which is a mandatory requirement for computational modeling.

\section{Patient-Specific Hemodynamics Computation}

In order to simulate the hemodynamics using a comprehensive heart model like the one presented in the previous section, the CFD solver must be able to handle the large deformations of the non-manifold heart surface, including multiple topological changes like valve closure. Such constraints pose difficulties for body-fitted grid methods like the finite element method, requiring extra effort for frequently re-meshing the whole domain and also adversely impacting the robustness and accuracy of the linear solver. To address these difficulties, we use in this work a level-set-based embedded boundary method 4]. The non-manifold heart polygonal mesh is embedded in a computational box endowed with a regular grid and the Navier-Stokes equations are solved inside the rectangular domain using finite difference and finite volume discretizations. The liquid inside the box and "outside" the left heart plays the role of the body circulatory system, whose flow resistance is simulated by imposing no slip boundary conditions on the sides of the box. The heart polygonal mesh is represented on the regular grid with spatial resolution $d x$ by defining the level set $\phi(x)=\operatorname{dist}(x$, mesh $)-d x$, and using it appropriately for defining the numerical stencils at the blood/tissue interface.

We solve the 3D Navier-Stokes equation for incompressible flow with viscous terms. Blood density and dynamic viscosity are set to $\rho_{\text {liquid }}=1.05 \mathrm{~g} / \mathrm{cm}^{3}$ and $\mu=0.003 \mathrm{~Pa} \cdot s$, respectively. The velocity of the mesh walls, extrapolated in space to the grid nodes and interpolated in time between two consecutive mesh

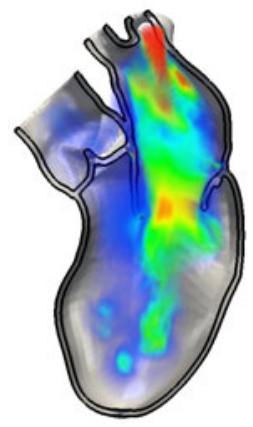

(a) (a)

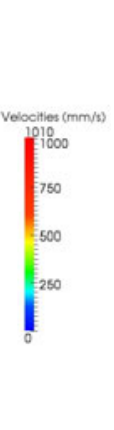

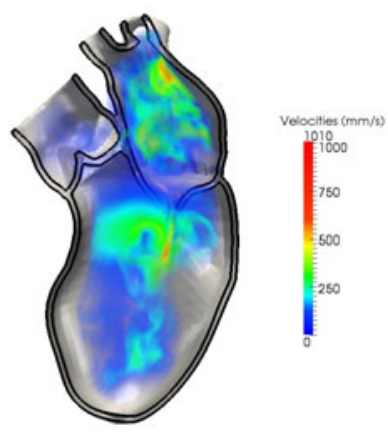

(b)

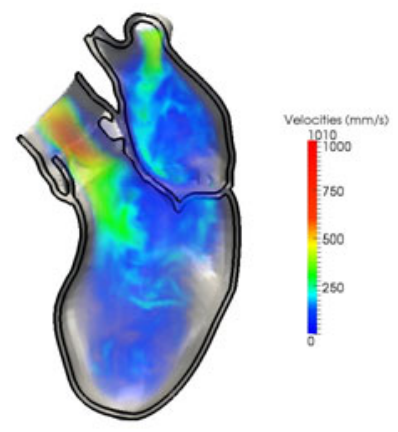

(c)

Fig. 2. Velocity magnitudes of the patient-specific simulated hemodynamics in early-diastole (a), late-diastole (b), and mid-systole (c) 
positions, are used to enforce no-slip conditions to the Navier-Stokes solver. We use the ghost fluid method to extend the velocity in the solid regions, when necessary. The convective solver relies on high-order Courant-Isaacson-Rees (CIR) techniques, while the viscous terms are treated semi-implicitly as in 9 . An efficient multi-grid preconditioned conjugate gradient solver is used to solve the pressure Poisson equation.

In our experiments, the computations were performed on grids with an isotropic cell resolution of $1 \mathrm{~mm}^{3}$ (i.e. $d x=1 \mathrm{~mm}$ ), which is in the same range as the TEE data resolution. The time step was chosen to obey the CourantFriedrichs-Lewy (CFL) condition $d t * \max (u)<d x$, which enforces that information carried by the blood velocity $u$ does not travel faster than one grid cell per time step. The result of the computational fluid dynamics simulation is illustrated for a specific patient in Fig. 2, For further details the reader is referred to 4 .

\section{Validation Methodology}

We introduce a novel methodology for validation of simulated heart hemodynamics with clinically acquired Doppler measurements. Doppler echocardiography is routinely performed during cardiac exams to determine blood velocities from the phase shift between emitted and reflected high frequency ultrasound waves 1011. We address both, continuous wave (CW) Doppler and pulse wave (PW) Doppler, which measure velocities along the probe direction to produce $1 \mathrm{D}$ signals. For a conclusive comparison we reconstruct similar $1 \mathrm{D}$ signals from the simulated 4D CFD velocities.

CW returns the velocities of all blood cells along the probe path. The outer envelope of the signal corresponds to the maximum velocity. $\mathrm{CW}$ is used in practice to acquire the very high speed of regurgitation flows, however without providing the spatial location of the measurement. In the $4 \mathrm{D}$ patient-specific computational model, a virtual CW probe path is defined to match the real position and orientation of the corresponding ground truth Doppler. The 4D velocities are projected onto the probe direction for the entire cardiac cycle. Then, for each cardiac phase, the maximum velocity values along the probe path are registered. Velocities are sampled in $1 \mathrm{~mm}$ spatial steps along the probe path and at each location averaged over a small disk of radius $1.5 \mathrm{~mm}$, to realistically match the resolution of the CW protocol (Fig. 3 Top panel).

PW returns the dominant velocity of the blood cells inside a focal region of interest (ROI) along the probe direction, typically just below the mitral leaflet tips or within the left ventricular outflow tract [1011] (Fig. 3 Bottom panel). PW Doppler is used to measure the blood inflow and outflow across the valves and is limited in capturing high velocities. In the 4D patient-specific computational models, a virtual PW probe direction and ROI is defined to match the real position and orientation of the corresponding ground truth Doppler (Fig. 3 Bottom left). The $4 \mathrm{D}$ computed velocities inside the ROI are projected along the probe direction. The dominant velocity of the blood cells is approximated 


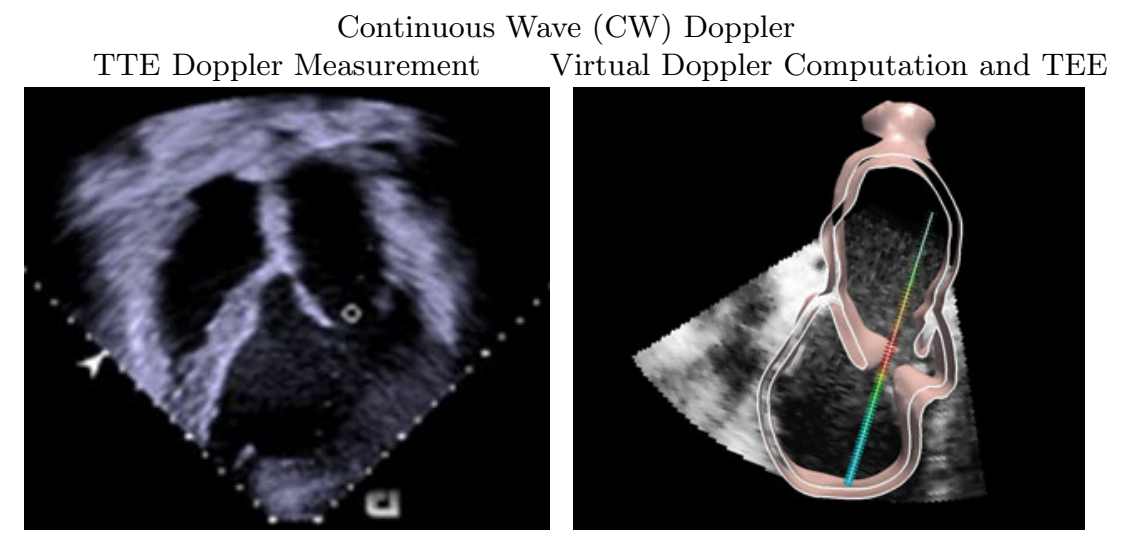

Pulse Wave (PW) Doppler

TTE Doppler Measurement

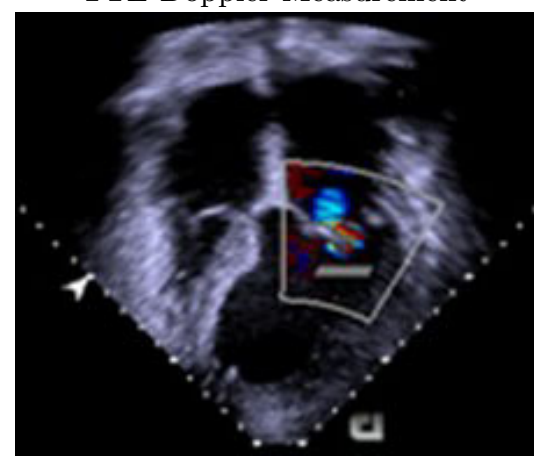

Virtual Doppler Computation and TEE

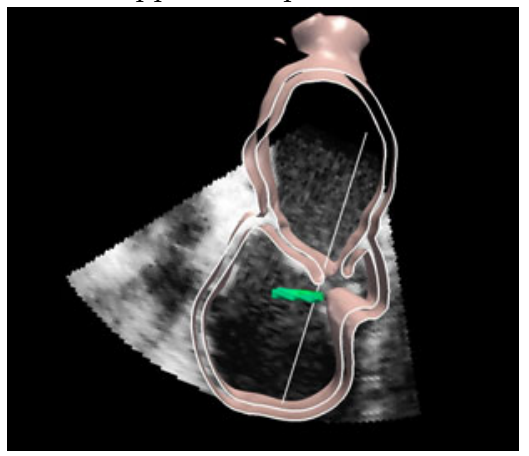

Fig. 3. Validation setup. CW and PW Doppler are computed from the 4D simulation (right panels) to reproduce the 1D ground truth (left panels).

by the most frequent velocity found in the ROI, determined automatically using a histogram. In our experiments, the number of points in the ROI vary between 300 and 700, while 10 bins were used to build the histogram.

While we focused in our experiments on transvalvular blood velocities as routinely measured during Doppler exams, it is important to note that the technique is not limited to these particular sites, and is applicable at any location.

\section{Results}

The performance of the patient-specific anatomy and dynamics computation was validated on a set of 239 patients with $4 \mathrm{D}$ echocardiography acquisitions. On average the precision was $1.73 \mathrm{~mm}$ at a speed of $4.8 \mathrm{sec}$ per volume for the valvular model and $2.68 \mathrm{~mm}$ at a speed of less than $1 \mathrm{sec}$ per volume for the left ventricle 67 .

The validation of our patient-specific hemodynamics computation - the main focus of this paper - was performed against clinical relevant blood flow 


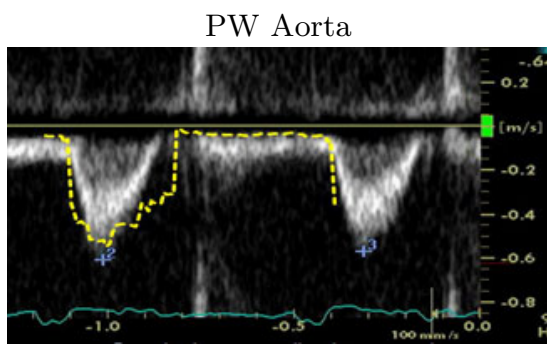

PW Mitral

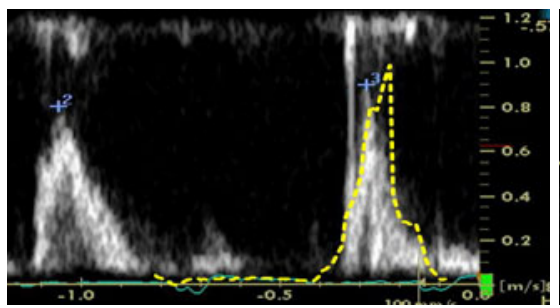

CW Aorta

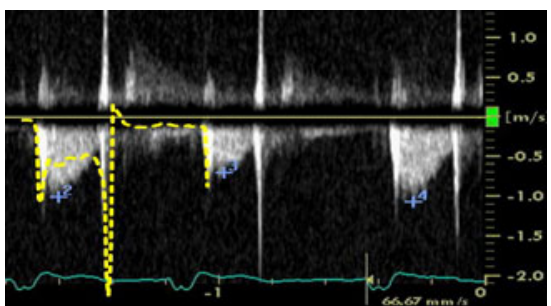

CW Mitral

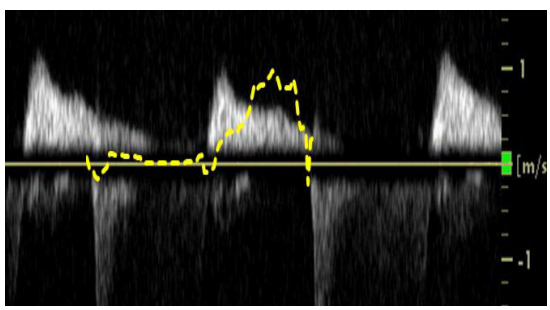

Fig. 4. Doppler acquisitions overlaid with computed curves in a patient with atrial fibrillation. Note the absence of the A-Wave in the transmitral measurements (bottom row) and the high velocity peaks in the $\mathrm{CW}$ aortic measurements (top right), which were well captured by the simulation.

Table 1. Measured ground truth (M) and simulated (S) peak velocities. Patient 1 and 2 had atrial fibrillation, therefore the A-wave was not visible (symbolized by $\mathrm{n} / \mathrm{a}$ ) in the ground truth, also successfully captured by the simulation.

\begin{tabular}{r|c|c|c|c|c|c} 
& \multicolumn{2}{|c|}{ Patient 1 } & Patient 2 & Patient 3 \\
\hline peak velocity (m/s) & $\mathrm{S}$ & $\mathrm{M}$ & $\mathrm{S}$ & $\mathrm{M}$ & $\mathrm{S}$ & $\mathrm{M}$ \\
\hline Aortic PW systolic & 0.627 & 0.732 & 1.01 & 1.38 & 0.51 & 0.59 \\
Aortic CW systolic & 0.761 & 0.843 & 1.11 & 1.53 & 1.035 & 0.82 \\
Mitral PW diastolic E-Wave & 0.78 & 0.8 & 0.49 & 0.48 & 0.97 & 0.853 \\
Mitral PW diastolic A-Wave & n/a & 0.53 & 0.4 & \multicolumn{2}{|c}{$\mathrm{n} / \mathrm{a}$} \\
Mitral CW diastolic & 0.96 & 1.0 & 0.72 & 0.8 & 1.01 & 1.05
\end{tabular}

measurements, routinely acquired using Doppler echocardiography techniques. It is important to notice that Doppler methods, such as the aortic CW Doppler for stenosis assessment, are the current gold standard for hemodynamic analysis. Please also note that the diagnostic value of Doppler measurements is to a large extent in the peak velocities - as e.g. for assessing the degree of dysfunction - as well as qualitative observations in particular modes, i.e. the E- and A-waves in Mitral PW Doppler, describing transmitral flow early and late diastole respectively. Hence the evaluation is focused on these aspects.

The patient population included three randomly selected patients: two of them suffering from atrial fibrillation whereas the third from severe regurgitations of both, aortic and mitral valves. For each patient, a Doppler exam was performed six to 12 weeks before surgery, at the beginning of which $4 \mathrm{D}$ TEE data was 
acquired. A difference in heart rates of 1 to $18 \%$ could be observed. Patientspecific models of anatomy and dynamics were computed form the 4D TEE images as described in section 2 From the obtained models, boundary conditions were derived and used to computed patient-specific hemodynamics as presented in section 3. Using the validation protocol described in section 4, measured and computed Doppler (PW and CW), for both valves and all patients, were compared and reported in Table 1. Overall a high agreement could be observed between the in vivo acquired measurements and computed results and the correlation among the corresponding values, which was computed using all values from all patients as reported in Table 1, amounted to $r=0.85$ (significance $p<0.0002675)$. A qualitative comparison is provided in Fig. 4, which displays the ground truth Doppler velocities over time and the computed curves in one patient with atrial fibrillation. In support of our quantitative comparison, the overlay reveals the high level of detail of our computational model. In particular, the absence of A-wave in the transmitral measurements as specific for atrial fibrillation was correctly captured by the model - whereas its presence reflects ventricular filling in late diastole. Moreover, sharp peaks observed in CW Doppler across aortic valve were reproduced by the simulation, which occur across the valve orifice just before closure and after opening.

\section{Conclusion}

This paper presents a framework for systemic and highly detailed modeling of left heart anatomy, dynamics and hemodynamics from 4D TEE. Fast and robust estimation of anatomy and dynamics is performed using machine learning algorithms, while patient-specific hemodynamics is robustly estimated by using the personalized left heart model as a boundary condition for solving a level-set based formulation of the Navier-Stokes equations. Our framework was validated on three diseased patients, where both TEE and Doppler measurements were available and demonstrated high agreement. To the best of our knowledge our personalized model captures the most anatomical detail, considering for the first time papillary muscles and importantly is the first time that a framework for cardiac fluid mechanics is validated with clinically acquired ground truth data obtained from routinely employed clinical instrumentation in diseased patients.

\section{References}

1. Chung, E., Leon, A., Tavazzi, L., Sun, J., Nihoyannopoulos, P., Merlino, J., Abraham, W., Ghio, S., Leclercq, C., Bax, J., Yu, C., Gorcsan, J., Sutton, S., De Sutter, J., Murillo, J.: Results of the Predictors of Response to CRT (PROSPECT) Trial. Circulation (117), 2608-2616 (2008)

2. Hunter, P., Coveney, P., de Bono, B., Diaz, V., Fenner, J., Frangi, A., Harris, P., Hose, R., Kohl, P., Lawford, P., McCormack, K., Mendes, M., Omholt, S., Quarteroni, A., Skar, J., Tegner, J., Randall, T.S., Tollis, I., Tsamardinos, I., van Beek, J., Viceconti, M.: A vision and strategy for the virtual physiological human in 2010 and beyond. Phil. Trans. R. Soc. A 368(1920), 2595-2614 (1920) 
3. Mcqueen, D.M., Peskin, C.S.: A three-dimensional computer model of the human heart for studying cardiac fluid dynamics. SIGGRAPH 34(1), 56-60 (2000)

4. Mihalef, V., Metaxas, D., Sussman, M., Hurmusiadis, V., Axel, L.: Atrioventricular blood flow simulation based on patient-specific data. In: Ayache, N., Delingette, H., Sermesant, M. (eds.) FIMH 2009. LNCS, vol. 5528, pp. 386-395. Springer, Heidelberg (2009)

5. Schenkel, T., Malve, M., Reik, M., Markl, M., Jung, B., Oertel, H.: MRI-Based CFD Analysis of Flow in a Human Left Ventricle: Methodology and Application to a Healthy Heart. Annals of Biomedical Engineering (3), 503-515 (2007)

6. Ionasec, R.I., Voigt, I., Georgescu, B., Wang, Y., Houle, H., Vega-Higuera, F., Navab, N., Comaniciu, D.: Patient-specific modeling and quantification of the aortic and mitral valves from $4 \mathrm{D}$ cardiac CT and TEE. IEEE Transactions on Medical Imaging 9, 1636-1651 (2010)

7. Wang, Y., Georgescu, B., Houle, H., Comaniciu, D.: Volumetric myocardial mechanics from $3 \mathrm{D}+\mathrm{t}$ ultrasound data with multi-model tracking. In: Camara, O., Pop, M., Rhode, K., Sermesant, M., Smith, N., Young, A. (eds.) STACOM 2010. LNCS, vol. 6364, pp. 184-193. Springer, Heidelberg (2010)

8. Zheng, Y., Barbu, A., Georgescu, B., Scheuering, M., Comaniciu, D.: Four-chamber heart modeling and automatic segmentation for 3D cardiac CT volumes using marginal space learning and steerable features. IEEE Transactions on Medical Imaging 27(11), 1668-1681 (2008)

9. Li, J., Renardy, Y., Renardy, M.: Numerical simulation of breakup of a viscous drop in simple shear flow through a volume-of-fluid method. Phys. Fluids 12(2), 269-282 (2000)

10. Quinones, M., Otto, C., Stoddard, M., Waggoner, A., Zoghbi, W.: Recommendations for Quantification of Doppler Echocardiography: A Report from the Doppler Quantification Task Force of the Nomenclature and Standards Committee of the American Society of Echocardiography. J. Am. Soc. E (15), 167-184 (2002)

11. Armstrong, W., Ryan, T.: Feigenbaums Echocardiography. Lippincott Williams and Wilkins (2009) 